\title{
Low-frequency ultrasound can drive the transport of nanoparticles and molecules in polymer gels for biotechnology applications
}

\author{
Donald K. Martin
}

\begin{abstract}
This paper reports the use of low-frequency ultrasound to influence transport in porous hydrogels with a transducer attached in direct contact with the hydrogel. This is a different configuration than for ultrasound-generating devices utilized previously for enhancing transport of molecules. The advantages of the system reported in this manuscript are that (i) much less acoustic power is required to influence the transport in the hydrogel that is in direct contact with the ultrasonic transducer, and (ii) no cavitation is induced in the hydrogel to influence the transport. This system was first tested in bench-top in vitro experiments by quantifying the transport of gold nanoparticles stimulated by low-frequency ultrasound. Then, to provide an in vivo example for potential biotechology applications, the system was demonstrated to be capable of transporting drugs across the tunics of a rabbit eye into the ocular circulation so as to target the transported drug to the outer retina.
\end{abstract}

Keywords: Gold nanoparticles, agarose gel, drug delivery, low-frequency ultrasound

Univ. Grenoble Alpes, CNRS, Grenoble INP, TIMC-IMAG / SyNaBi (UMR 5525), 38000 Grenoble, France.

Corresponding author: D. K. Martin E-mail: don.martin@univ-grenoble-alpes.fr

DOI: 10.2478/ebtj-2019-0001

(C) 2019 Authors. This work was licensed under the Creative Commons AttributionNonCommercial-NoDerivs 4.0 License.

\section{Introduction}

Techniques for externally stimulating the release and diffusion of drugs from polymeric drug delivery systems enable finer tuned control of drug delivery for selective treatments. For example, the external stimulus can be triggered when the delivery system is placed close to the targeted delivery site or reaches an appropriate environment. For some time ultrasound has been investigated as a useful external stimulus for enhancing mass-transport of drugs (1). Most of the previous reports of ultrasound-stimulated drug delivery investigate an acoustic field generated by an ultrasonic horn located remotely from the drug-containing polymer (2), or indeed have considered the use of ultrasound to enhance transport across barriers such as the skin (3).

A low-frequency ultrasonic horn is a common device but with a complex operating mode. The most common usage of such ultrasonic horns is to generate $20-30 \mathrm{kHz}$ continuous-wave fields into water or an aqueous solution that has undergone no special treatment (such as degassing or deionizing) under atmospheric pressure (4). For such conditions the threshold acoustic pressure to generate inertial cavitation is around $100-120 \mathrm{kPa}$ (zero-to-peak amplitude). As the driving pressure exceeds those values, a relatively broad range of initial bubble sizes (from microns to tens of microns radius) will generate inertial cavitation (4). The direct sound field that is generated from the immersed tip of the ultrasonic horn has an amplitude that decreases rapidly with distance from the tip. Even in the absence of cavitation, the overall sound field in the liquid itself is a combination of this direct field and that generated by the reverberation and reflections from walls of the vessel which contain the liquid (5).

Ultrasonic horns immersed in aqueous fluids have been reported to stimulate, remotely, drug-release from polymers. For a recently published example, the application of ultrasound using an immersed transducer held remotely at a distance of $1.5 \mathrm{~cm}$ enhanced 
release of fluorescein from poly(lactic acid-co-glycolic acid), PLGA, implants that were injected into tissue-mimicking hydrogel phantoms (acrylamide, $2 \mathrm{kPa}$ elastic modulus) (6). However, in that example, ultrasound at $3 \mathrm{MHz}$ and only at an intensity of $2.2 \mathrm{~W} / \mathrm{cm}^{2}$, but not at $0.7 \mathrm{~W} / \mathrm{cm}^{2}$, was capable to enhance release of fluorescein with a concomitant induced degradation of the PLGA. Ultrasound of $43 \mathrm{kHz}$ was applied remotely at a distance of $3.7 \mathrm{~cm}$ to cellulose hydrogels, and this was reported to enhance the release of a drug (mimosa) but with the ultrasound power from 5 to $30 \mathrm{~W}$ (7).

The mechanism for such ultrasound-stimulated release, particularly for transport of molecules across the skin barrier, is usually attributed to inertial cavitation since the intensities of the ultrasound at which maximum enhanced release occurred were about $14 \mathrm{~W} / \mathrm{cm}^{2}$ for $20 \mathrm{kHz}$ and $17 \mathrm{~W} / \mathrm{cm}^{2}$ for $40 \mathrm{kHz}(3)$, which are above the cavitation threshold power. Such intensities also have the potential for damage to surrounding materials and tissues, since it has been reported that the intensities at which maximum enhancement of skin permeability was induced by low-frequency ultrasound $\left(14 \mathrm{~W} / \mathrm{cm}^{2}\right.$ for $20 \mathrm{kHz}$ and $17 \mathrm{~W} / \mathrm{cm}^{2}$ for $40 \mathrm{kHz}$ ) also caused pitting damage of aluminum foil due to the cavitation (3). Indeed, intensities of low-frequency ultrasound $(23 \mathrm{kHz})$ above the cavitation threshold degraded aqueous polyvinyl alcohol (PVA) polymer (8).

This paper reports the use of low-frequency ultrasound to influence transport in porous hydrogels with a transducer attached in direct contact with the hydrogel. This is a different configuration than for ultrasound-generating devices utilized previously for enhancing transport of molecules. The advantages of the system reported in this manuscript are that (i) much less acoustic power is required to influence the transport in the hydrogel that is in direct contact with the ultrasonic transducer, and (ii) no cavitation is induced in the hydrogel to influence the transport. This system was first tested in bench-top in vitro experiments by quantifying the transport of gold nanoparticles stimulated by low-frequency ultrasound. Then, to provide an in vivo example for potential biotechology applications, the system was demonstrated to be capable of transporting drugs across the tunics of a rabbit eye into the ocular circulation so as to target the transported drug to the outer retina.

\section{Methods}

In vitro experiments to confirm physical mechanism of ultrasound-stimulated delivery

An apparatus was designed to provide a direct contact between a low-frequency ultrasound transducer and a hydrogel that contained a tracer nanoparticle (Fig. 1). The low-frequency ultrasound was stimulated by a signal generator $(40 \mathrm{kHz}$ at 0.2 $\mathrm{W} / \mathrm{cm}^{2}$, marked as 5 in Fig. 1). The hydrogel was formed by dissolving agarose $(0.5 \% \mathrm{w} / \mathrm{v})$ in MilliQ water at a temperature above the gelling point, pouring the agarose solution into a mould and then allowing the solution to cool below the gelling temperature. The tracer gold nanoparticles $(15-20 \mathrm{~nm})$ were added to the agarose solution before it was allowed to cool below the gelling temperature. The gel formed with the incor- porated gold nanoparticles was called the "tracer-gel" and is marked as 2 in Fig. 1. Another agarose gel was formed without adding any nanoparticles and this was called the "receiver-gel" and is marked as 3 in Fig. 1. The "tracer-gel" was firmly contacted to the ultrasound transducer (marked as 1 in Fig. 1). The combination of the ultrasound transducer, "tracer-gel" and "receiver-gel" were supported by a microscope slide (Fig. 1).

The diffusion of the tracer gold nanoparticles was quantified by measuring the change in transmittance of the "receiver-gel" that was firmly contacted to the "tracer-gel". The change in transmittance was measured by recording the output voltage of a photodiode (marked as 7 in Fig. 1) upon which the beam from a HeNe laser was directed (marked as 6 in Fig. 1). For example, the output voltage from the photodiode would decrease if the transmittance of the "receiver-gel" was reduced due to the accumulation of the tracer gold nanoparticles. The transmittance of the "receiver-gel" was measured at a known distance from the interface at the "tracer-gel" (marked as $\mathrm{x}$ in Fig. 1). In the experiments, the transmittance was measured at the distances of $\mathrm{x}=1 \mathrm{~mm}$ and $\mathrm{x}=2 \mathrm{~mm}$. The transmittance data were captured by a computer (marked 8 in Fig. 1).

\section{In vivo experiments to test the ultrasound-induced delivery of therapeutic molecules}

The principle of the in vitro system was tested by placing the "tracer-gel" in direct contact with the conjunctiva and sclera at the limbal region of the eye of a rabbit. In effect, the tissues at the limbal region substituted for the "receiver-gel". The "tracer-gel" included therapeutic molecules that would usually have been injected directly into the vitreous humor of the eye in order for diffusion to the retina. The thickness of the tissues at the limbal region of the eye is around $0.5 \mathrm{~mm}$ or less, so the hypothesis was that the ultrasound would drive the therapeutic molecules through the conjunctiva and sclera to be delivered to the capillary circulation of the uveal tract in the region of the pars plana. The uveal circulation would then deliver the therapeutic molecules to the interior of the eye, including to the retina.

Therapeutic molecules were loaded into the hydrogel ("tracer-gel") that was attached to the ultrasound transducer. The therapeutic drugs replaced the gold nanoparticles that were used for the in vitro experiments. The therapeutic molecules were bevacizumab $(25 \mathrm{mg} / \mathrm{ml})$, ranibizumab $(3 \mathrm{mg} / \mathrm{ml})$ and verteporfin $(2 \mathrm{mg} / \mathrm{ml})$ either incorporated directly into the agarose "tracer-gel" or encapsulated in liposomes that were then incorporated into the agarose "tracer-gel". Each drug was incorporated in the "tracer-gel" at the concentration used for the current clinical procedure of direct injection into the vitreous chamber (bevacizumab, ranibizumab) or IV injection (verteporfin).

\section{(a) Encapsulation of drug into liposomes}

The principle was to prepare a dried film of lipid, rehydrate the lipid in a buffer containing the drug to be packaged and then produce homogeneous liposomes of $1 \mu \mathrm{m}$ diameter using an extrusion procedure. The result of that procedure was a $1 \mathrm{ml}$ 

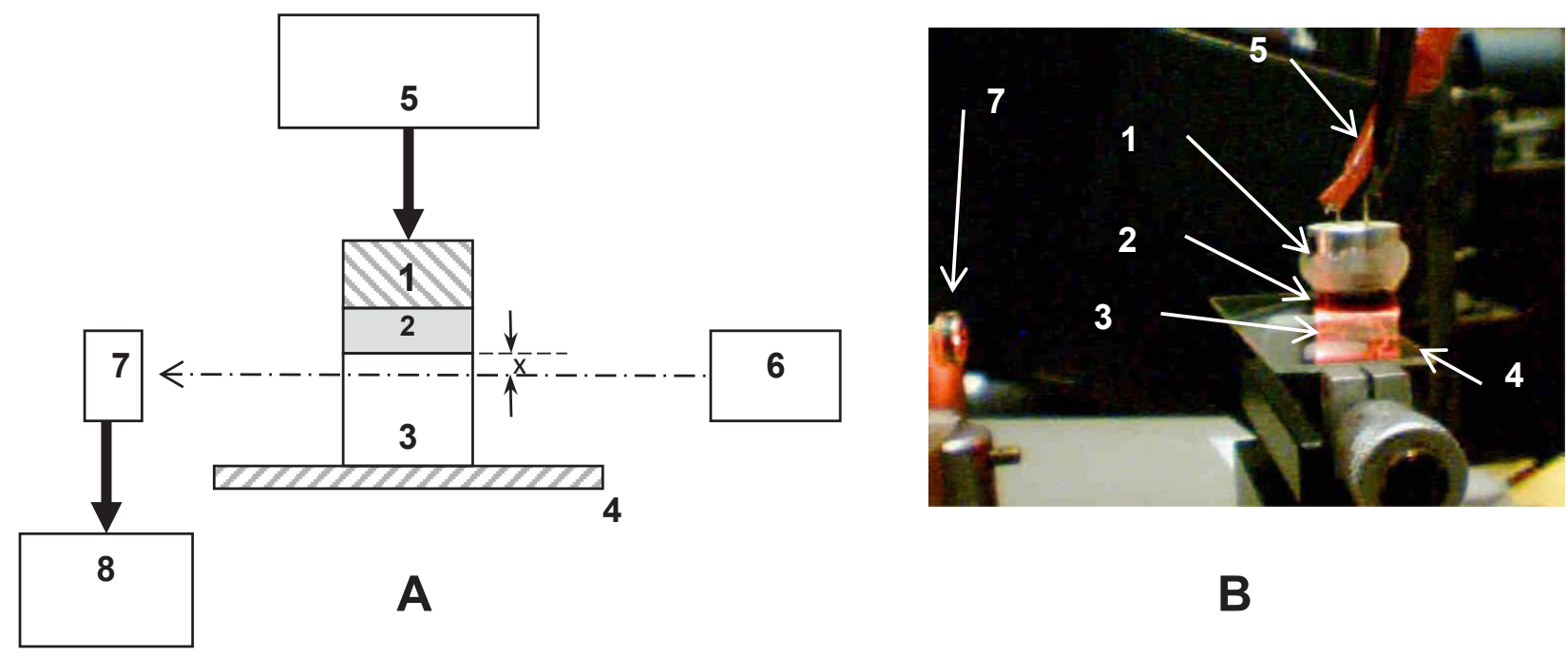

B

Figure 1. Apparatus used to measure the transport of tracer gold nanoparticles that as stimulated by low-frequency ultrasound. The block diagram of the components is drawn in panel A, and the actual gels+transducer is shown in panel B. The light path is illustrated with the dotted line, and was aimed to pass through the "receiver-gel" at a distance marked $\mathrm{x}$ below the interface with the "tracer-gel". The numbers in panels A and B refer to the same components. (1=ultrasonic transducer, 2="tracer-gel", 3="receiver-gel", 4=glass microscope slide, 5=signal generator, 6=HeNe laser, 7=photodiode, 8=computer).

volume of a concentrated solution of liposomes of $1 \mu \mathrm{m}$ diameter that were loaded with the drug. This was achieved using the following procedure. A quantity of $40 \mu \mathrm{g}$ of L- $\alpha$-lecithin (Sigma, $\mathrm{P}-5638$ ) was added to a glass tube and $400 \mathrm{ml}$ of chloroform was added to the glass tube in order to achieve a lipid concentration of $100 \mathrm{mg} / \mathrm{ml}$ and vortex mixed to ensure the lipid was completely dissolved. The lipid/chloroform mixture was dried under a stream of $\mathrm{N}_{2}$ gas to remove the volatile chloroform. The glass tube was left under vacuum for at least 12 hours at room temperature in order to remove all traces of chloroform. Then a $1 \mathrm{ml}$ solution of the therapeutic drug, at the required concentration, was addd to the glass tube and vortex mixed for 5 minutes to ensure complete solution of the lipid. The tube was left to stand at room temperature for at least 15 minutes, then vortex mixed again for 5 minutes. This mixture was transferred to a syringe for extrusion through a $1 \mu \mathrm{m}$ polycarbonate filter 19 times in order to produce a homogeneous solution of liposomes. The liposome mixture was transferred to an Eppendorf tube and centrifuged at $9,000 \mathrm{rpm}$ for 30 minutes in order to pellet the liposomes, the supernatant was removed and the pellet resuspended in a clear $150 \mathrm{mM} \mathrm{NaCl}$ (unbuffered) solution. This final solution ws incorporated into the agarose "tracer-gel".

\section{(b) Ultrasound-induced delivery of therapeutic molecules to the limbal region of the eye}

The rabbits used for these experiments were female New Zealand White of $2-2.5 \mathrm{~kg}$ in weight. The rabbits were anaesthetised, prior to any procedure, using inhalational anaesthesia that comprised a mixture of $2.0-3.0 \%$ isoflurane with a combination of oxygen and nitrous oxide (in the ratio 2.5:1). The gas mixtures are delivered to the rabbit via a close-fitting nose-cone that was attached to a Stinger anaesthesia station. The concentration of isoflurane was varied within the range specified during the procedures in order to maintain optimal respiration for the rabbits and to maintain a surgical level of anaesthesia. All procedures were acute and the rabbits did not recover from anaesthesia. The animal care was humane and in accord with institutional guidelines procedures that were approved by the local Animal Ethics Authority.

After the depth of anaesthesia was confirmed by a lack of corneal and muscle reflexes, a lid-speculum was gently inserted to retract the eyelids of the rabbit. Where necessary due to a narrow palpebral aperture, the globe was exposed by incisions at the lateral and medial canthi, removing the nictitating membrane and reflecting the eyelids. The "tracer-gel" attached to the ultrasound transducer was placed in contact with the limbal region on the temporal side of the eye. The transducer was activated to deliver the $40 \mathrm{kHz}$ ultrasound for a period of 1-2 minutes. For each eye of 3 rabbits a different therapeutic molecule was tested, either incorporated directly into the agarose "tracer-gel" or encapsulated in liposomes that were then incorporated into the agarose "tracer-gel". Some eyes were not treated so as to be negative controls for the analyses of the eye tissues. In some eyes the therapeutic molecules were injected directly into the vitreous chamber to provide a positive control for the analyses of the eye tissues. After this period was completed for each eye, the rabbit was euthenased by $\mathrm{CO}_{2}$ asphyxiation, both eyes enucleated and immediately fixed in formalin for the subsequent fluorescent and immunohistochemical analyses.

\section{(c) Preparation of rabbit eye tissue for analysis}

The enucleated and fixed eyes were then embedded in paraffin and chilled on a cold plate at $-5^{\circ} \mathrm{C}$ for $15-30$ minutes. Thin sections ( $4 \mu \mathrm{m}$ thickness) were cut using a new disposable knife blade and floated on a water bath at $45-50^{\circ} \mathrm{C}$ while the paraffin-embedded eye remained on the 
cold plate. The floated sections were collected on ATS (3-aminopropyltriethoxy-silane) coated slides, which were then dried in an oven at $55^{\circ} \mathrm{C}$ for 1 hour. The sections were deparaffinized with the following sequence of solvents; xylene ( 1 minute, for each of two times), absolute alcohol (1 minute, for each of four times) and 70\% alcohol (1 minute, for one time).

\section{(d) Methods for analysis of eye sections}

The presence of bevacizumab or ranibizumab was detected with a published immunohistochemistry reaction (9) that used a sensitive and highly specific anti-human IgG antibody raised in donkey (Jackson ImmunoResearch Laboratories, cat\# 709165-149) to bind to either the bevacizumab or ranibizumab. The antibody was diluted in glycerol according to the manufacturer's specifications and stored at $-20^{\circ} \mathrm{C}$ until used. Stringent blocking was undertaken using donkey serum to minimise background binding of the antibody to VEGF receptor isotypes. The blocking solution consisted of PBS, donkey serum (1:20 dilution), BSA (0.5\%), Triton X-100 (0.1\%) and sodium azide $(0.05 \%)$. The tissue sections were incubated in the blocking solution overnight at $4^{\circ} \mathrm{C}$. The next day the antibody was further diluted 1:500 in the blocking solution, from the glycerol storage solution. The tissue sections were then incubated with the antibody overnight at $4^{\circ} \mathrm{C}$. The imaging of the stained sections was conducted using either a confocal microscope or a fluorescence microscope.

An antibody-binding reaction was unnecessary to detect the presence of verteporfin in the sections of the rabbit eye since verteporfin emits light in response to excitation at around $488 \mathrm{~nm}$. The emission peak is around $650 \mathrm{~nm}$ but has a broad tail. Thus, although the fluorescence microscope was optimised to detect FITC emissions (around 560nm) the intensity of the emission from verteporfin was sufficient for detection using a digital camera attached to a fluorescence microscope.

\section{Results}

In vitro experiments to confirm physical mechanism of ultrasound-stimulated delivery

This experiment was conducted in 2 parts with gold tracer nanoparticles incorporated in the "tracer-gel", which were (i) measurement of the transmittance in the "receiver-gel" at a distance of $\mathrm{x}=1 \mathrm{~mm}$ below the interface, and (ii) measure of the transmittance in the "receiver-gel" at a distance of $\mathrm{x}=2 \mathrm{~mm}$ below the interface. A negative control experiment was conducted to confirm whether changes in transmittance were due to transport of potential degradation products of the polymers as an explanation for any change in transmittance induced by the ultrasound. In that control experiment, the "tracer-gel" did not contain any gold nanoparticles and the transmittance in the "receiver-gel" was measured at a distance of $\mathrm{x}=1 \mathrm{~mm}$ below the interface. The interface is defined as the boundary between block 2 and block 3 in Fig. 1. In all these experiments the photodiode output $(\mathrm{mV})$ in the graphs indicates the transmittance. An increase in the transmittance of the gel corresponds to an increase in the photodiode output and a decrease in the transmittance of the gel corresponds to a decrease in the photodiode output.

The measured transmittance in the "receiver-gel" at $\mathrm{x}=1 \mathrm{~mm}$ below the interface is shown in Fig. 2. The diffusion of gold nanoparticles into the "receiver-gel" is measured by the change in transmittance. For example, we expect that an increase in the amount of gold nanoparticles diffusing into the clear gel would result in a decrease in the transmittance of the clear gel.

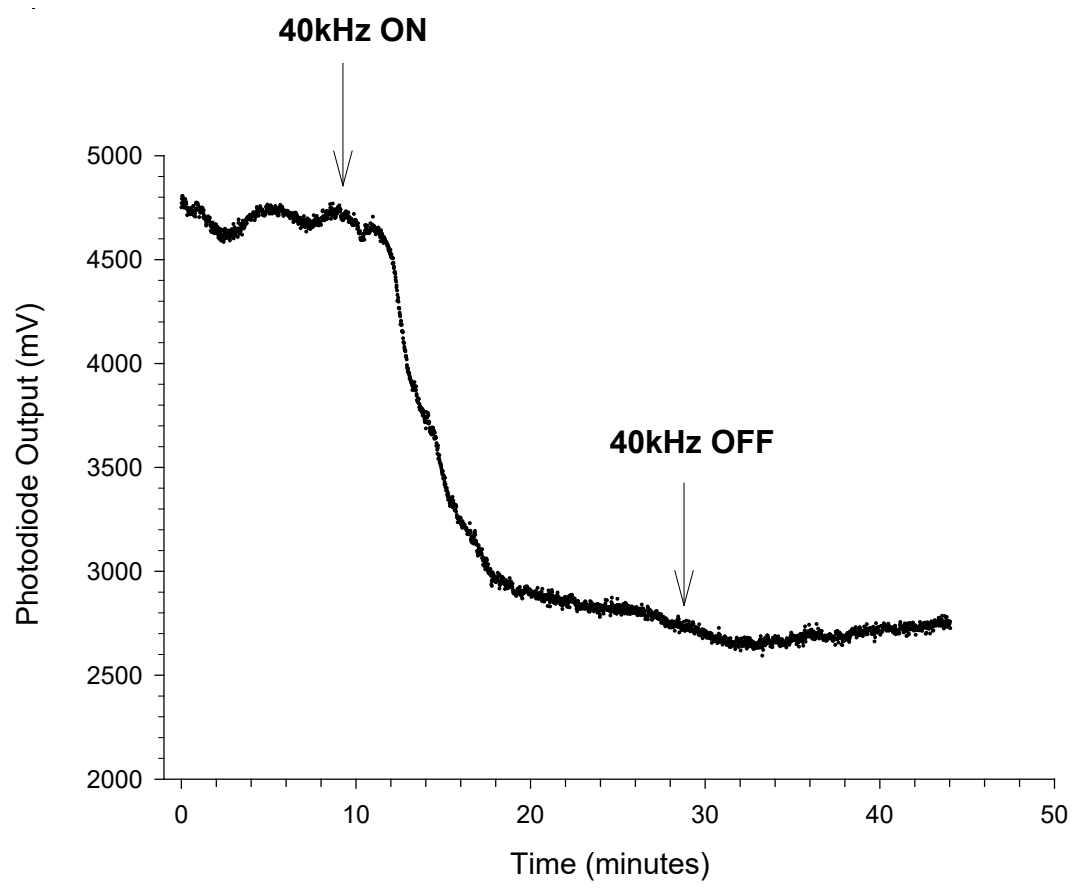

Figure 2. Measurement of transmittance of the "receiver-gel" at $x=1 \mathrm{~mm}$ depth from the interface with the "tracer-gel" that contained the gold nanoparticles. 


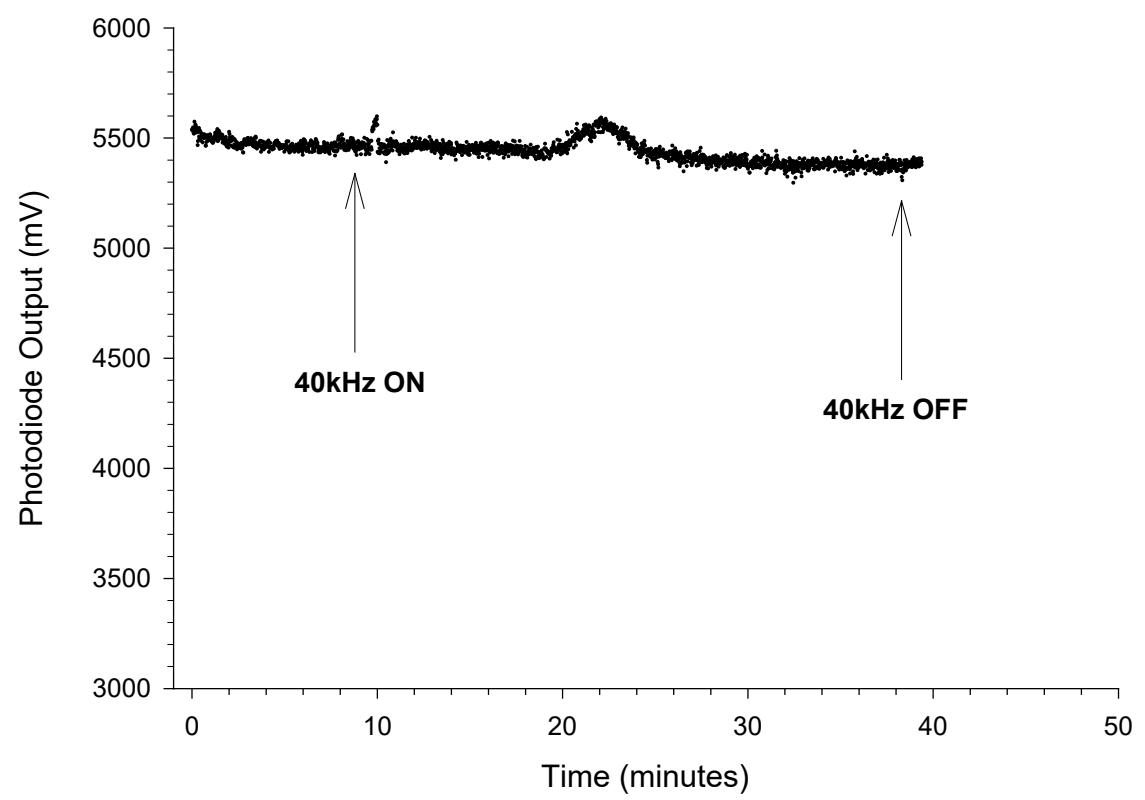

Figure 3. Measurement of transmittance of the "receiver-gel" at $x=2 \mathrm{~mm}$ depth from the interface with the "tracer-gel" that contained the gold nanoparticles.

Fig. 2 illustrates that the $40 \mathrm{kHz}$ ultrasound stimulated the diffusion of the gold nanoparticles from the "tracer-gel" into the "receiver-gel". There are 3 distinct regions of the data in Fig. 2: During the period from 0-10 minutes without the ultrasound switched on the transmittance of the "receiver-gel" was at a plateau level. The fluctuations may have been due to the initial setting up of the experiment. However, the most important feature is that the average level of transmittance was not changing. During the period from $10-30$ minutes the $40 \mathrm{kHz}$ ultrasound was switched on, as indicated by the arrows. The transmittance of the gel decreased (photodiode output decreased) soon after the $40 \mathrm{kHz}$ ultrasound was switched on. The decrease in transmittance was rapid at first, and then reached a plateau after about 18 minutes. The plateau phase continued until the $40 \mathrm{kHz}$ ultrasound was switched off. The plateau was probably due to the gold nanoparticles only being able to diffuse, under the stimulation of the ultrasound, for a limited distance into the clear gel. In this case, the distance was $1 \mathrm{~mm}$. This limit on diffusion can be explained by the mass of the gold nanoparticles, and comparison with measurements from techniques such as gel electrophoresis. For example, in gel electrophoresis the diffusion of substances is enhanced by an electrical current applied to a gel. A substance will diffuse more if it has a smaller mass, with substances of heavier mass diffusing less distance into the gel. By analogy, in the case of the $40 \mathrm{kHz}$ ultrasound the gold nanoparticles diffused as far into the "receiver-gel" as their mass would allow. During the period from 30-45 minutes the transmittance remained at the plateau level after the $40 \mathrm{kHz}$ ultrasound was switched off.

This limitation on how far the gold nanoparticle could diffuse into the gel is illustrated by the data shown in Fig. 3. The data in Fig. 3 are of the transmittance of the "receiver-gel" at a distance of $\mathrm{x}=2 \mathrm{~mm}$ below the interface. The transmittance in Fig. 3 does not change when the $40 \mathrm{kHz}$ was turned on. The small "bump" at around 20minutes is most likely due to an artefact. This lack of change in transmittance follows from the above explanation, whereby the mass of the gold nanoparticles is too great to allow the ultrasound energy at $40 \mathrm{kHz}$ to provide enough force (momentum) to enhance the diffusion of the gold nanoparticles further than about $1 \mathrm{~mm}$ into the "receiver-gel".

To support those interpretations of the experiment, the "receiver-gel" was imaged by scanning electron microscopy (SEM) and the particles that were observed in the SEM images were analysed by energy dispersive spectroscopy (EDS). Fig. 4 shows that the "receiver-gel" contained electron-dense particles. These particles are the lighter grey discrete objects resting on the underlying dehydrated gel. The location of these particles was at the surface of the dehydrated gel, which implied that the particles were at or just beneath the surface of the hydrated gel as it was used in the experiment.

Several of the particles in Fig. 4 appear to be quite large, and approximately $5 \mathrm{~mm}$ in size. This is much larger than the size of the gold nanoparticles used as the tracer. However, there are also smaller electron-dense particles in the clear gel, which are of the appropriate size (indicated by arrows in Fig 4). The chemical composition of all the particles was analysed by EDS. The larger particles contained $\mathrm{C}, \mathrm{O}, \mathrm{Na}, \mathrm{Mg}, \mathrm{Si}, \mathrm{Cl}, \mathrm{Ca}, \mathrm{Zn}, \mathrm{Fe}$ and $\mathrm{Cu}$. All of those chemicals are present in the buffer solutions used for the experiment. Thus, the larger particles are most likely crystals of the chemicals used for the solutions used to hydrate the gels.

Fig. 5 illustrates that the chemical composition of the smaller particles was gold. It is common for the gold nanoparticles to form clusters, which accounts for the size of the smaller particles as shown in Fig. 5.

A negative control experiment was conducted to confirm whether ultrasound-induced changes in transmittance could 


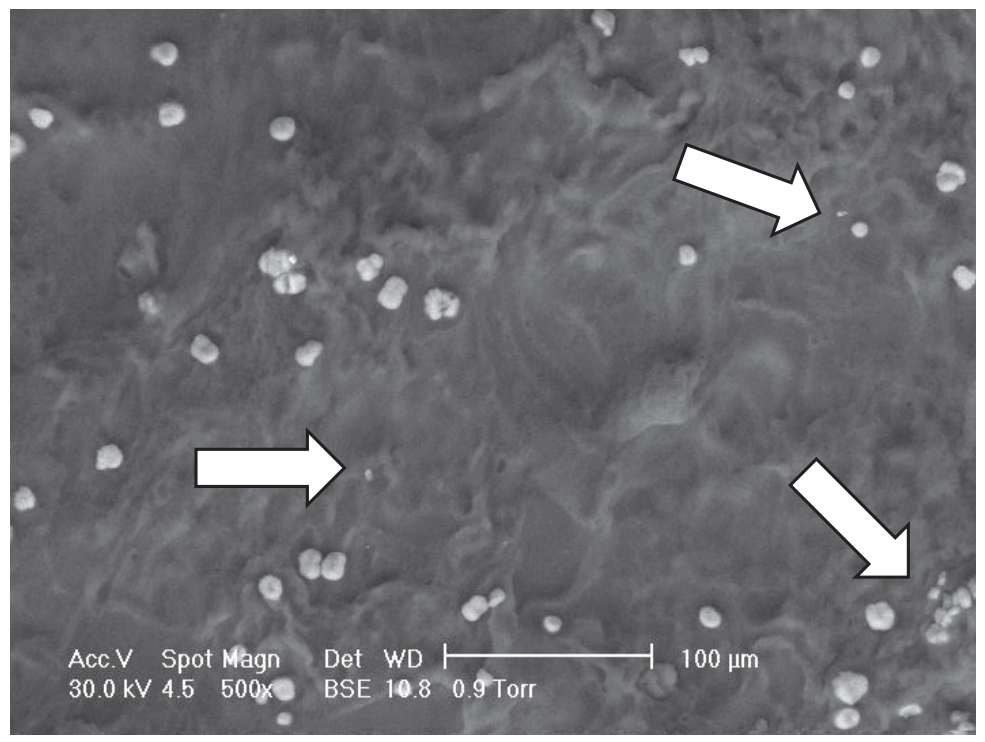

Figure 4. SEM image of the "receiver-gel" from the diffusion experiment shown in Fig. 1. For the purposes of the SEM imaging the gel was dehydrated. The electron-dense particles (light grey colour) are at the surface of the dehydrated gel. The arrows point to particles that are most likely to be gold, with the alrger particles most likely to be crystals of chemicals used in the buffer solutions (see text for explanation).

be explained by the transport of potential degradation products of the polymers. In that control experiment, the "tracer-gel" did not contain any gold nanoparticles and the transmittance in the "receiver-gel" was measured at a distance of $\mathrm{x}=1 \mathrm{~mm}$ below the interface. The results for this control experiment are shown in Fig. 6, which show that the transmittance of the "receiver-gel" did not change when measured at a distance of $\mathrm{x}=1 \mathrm{~mm}$ from the interface. This "receiver-gel" was analysed using SEM/EDS and no gold particles were found (data not shown).

This (negative) control experiment provided supporting evidence that the diffusion of the gold nanoparticles was enhanced by the $40 \mathrm{kHz}$ ultrasound and that the gold nanoparticles could only diffuse $1 \mathrm{~mm}$ into the "receiver-gel".

\section{A}

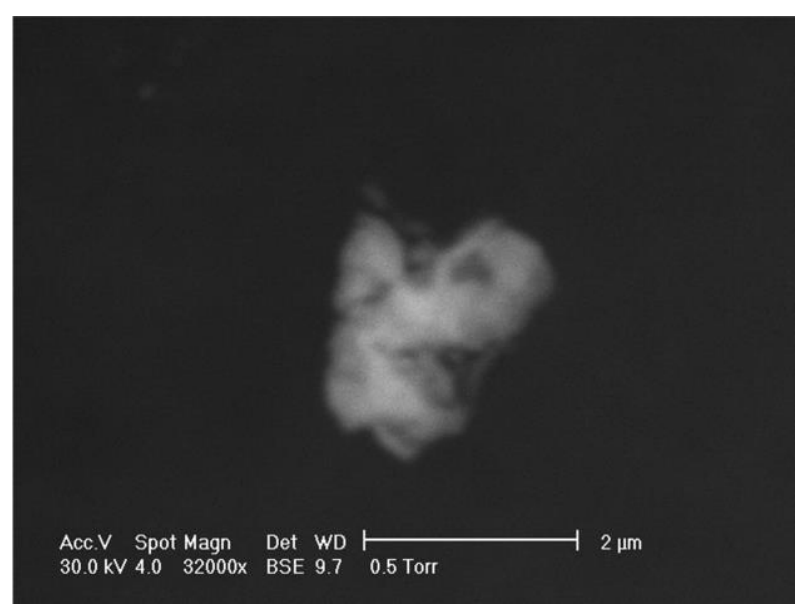

In vivo experiments to test the ultrasound-induced delivery of therapeutic molecules

Fig. 7 shows sections of an eye stained with Masson stain. The sections were from an eye into which bevacizumab was delivered from the "tracer-gel". The sections are useful for orientation, with collagen stained blue (cornea, sclera) and neural retina, epithelium and muscle stained crimson. The embedding and sectioning procedures have caused the retina to be dislodged from its normal position in contact with the sclera.

Fig. 8 shows sections of the retina around the posterior pole of the eyes of the 3 rabbits. The green fluorescence is not intense because of the very stringent blocking (donkey

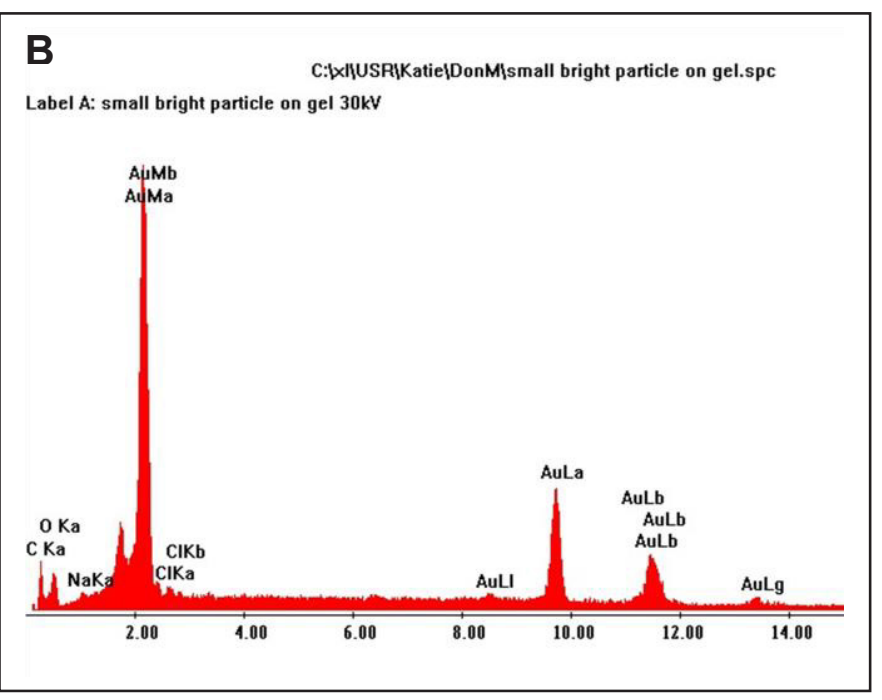

Figure 5. SEM/EDS analysis of the smaller particles observed in the dehydrated gels shown in Figure 4. The EDS analysis in panel $B$ is of the particle imaged by SEM in panel A. The analysis shows that the composition of the particle in panel A is gold. 


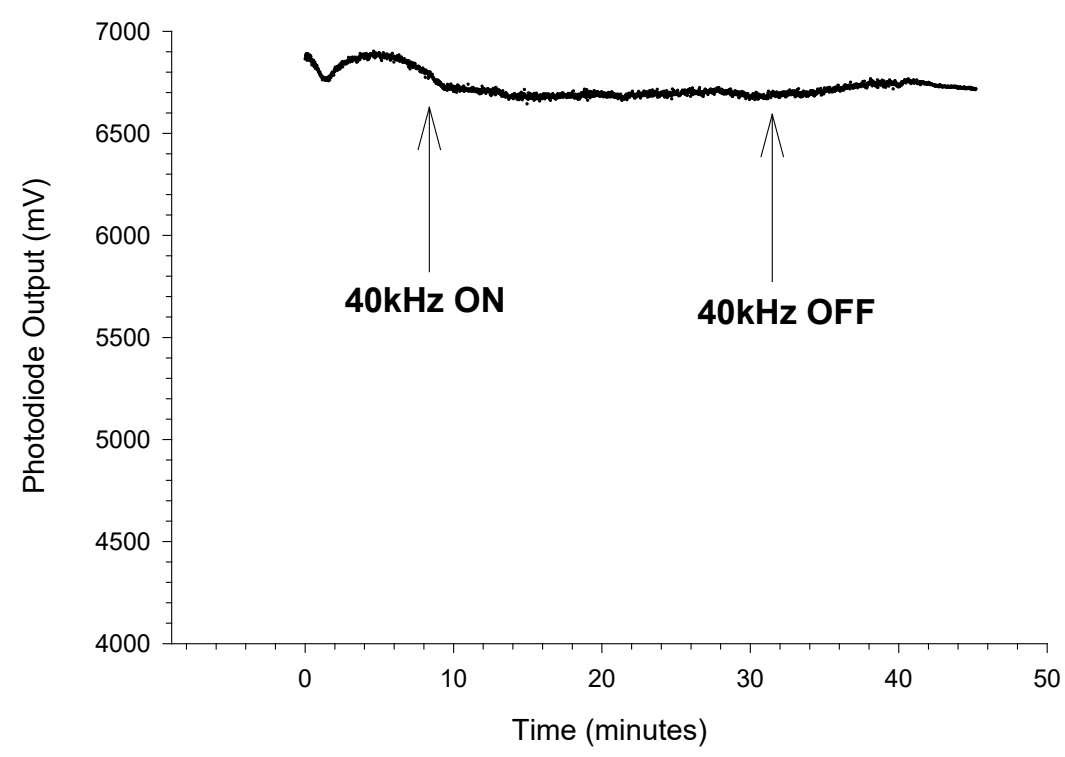

Figure 6. Measurement of transmittance of the "receiver-gel" at $x=1 \mathrm{~mm}$ depth from the interface with the "tracer-gel" that did not contain any gold nanoparticles.

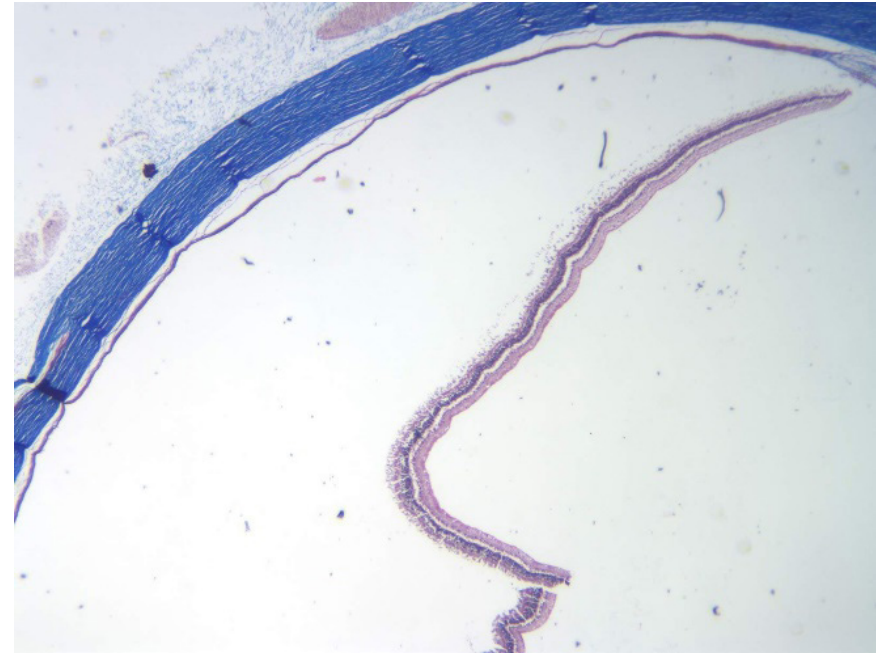

Figure 7A. Masson-stained section of a posterior area of a rabbit eye into which bevacizumab was delivered from the "tracer-gel". The sclera is stained bright blue and the retina, epithelia and muscle stained crimson. The retina and choroid have separated from the sclera.

serum, overnight at $4^{\circ} \mathrm{C}$ ) and slow antibody reaction (overnight at $4^{\circ} \mathrm{C}$ ) to minimise artefacts. The main point to note is the presence of the green staining in the outer retina and choriocapillaris in the images of eyes where bevacizumab (Fig. 8A, 8B), ranibizumab (Fig. 8D) and verteporfin (Fig. $8 \mathrm{~F}$ ) were delivered by the ultrasound stimulation. The outer retina is usually connected to the scleral tissue, but in these sections the retina had separated from the sclera. In comparison the negative control eye ("tracer-gel" with no drug) shows no green fluorescence (Fig. 8C). The positive control eye (direct injection into the vitreous chamber) shows green fluorescence (Fig. 8E).

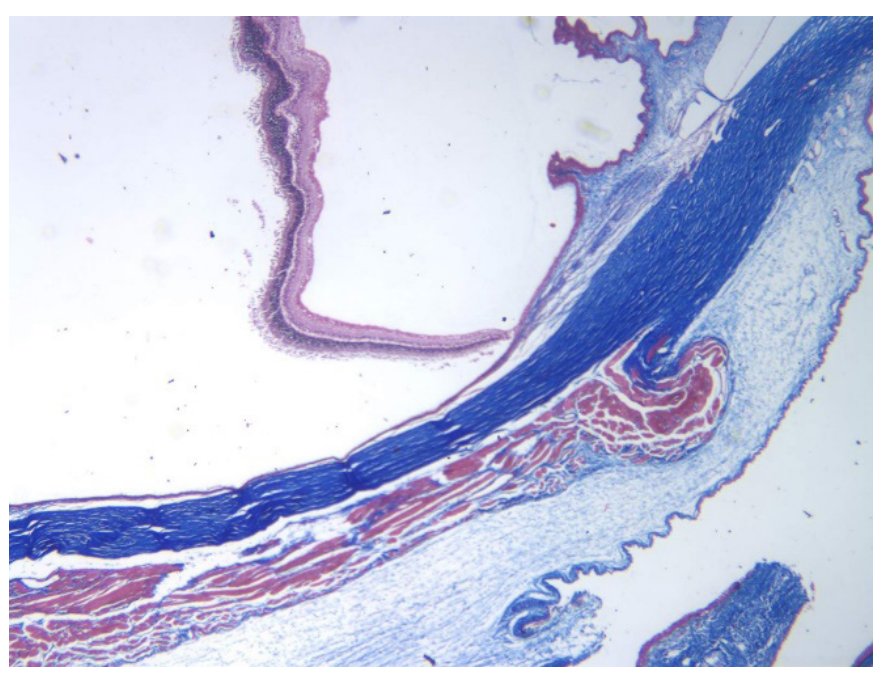

Figure 7B. Masson-stained section of the limbal region of a rabbit eye into which bevacizumab was delivered from the "tracer-gel". The sclera and cornea are stained bright blue and the retina, epithelia and muscle stained crimson. The retina and choroid have separated from the sclera.

\section{Discussion}

The results of these in vitro and in vivo experiments suggest that low-frequency ultrasound can be used to enhance the diffusion of nanoparticles and molecules in agarose gels. The use of low-frequency ultrasound to stimulate the release of molecules from polymers has been reported previously. However, in those previous reports the ultrasound transducer enhanced the release of molecules from polymers immersed in liquids and at a distance more than $1 \mathrm{~cm}$ remote from the ultrasound transducer $(6,7)$. Moreover, the power required for the enhanced release was usually more than $10 \mathrm{~W} / \mathrm{cm}^{2}$, since that level of power is required to be greater than the cavitation threshold, since 

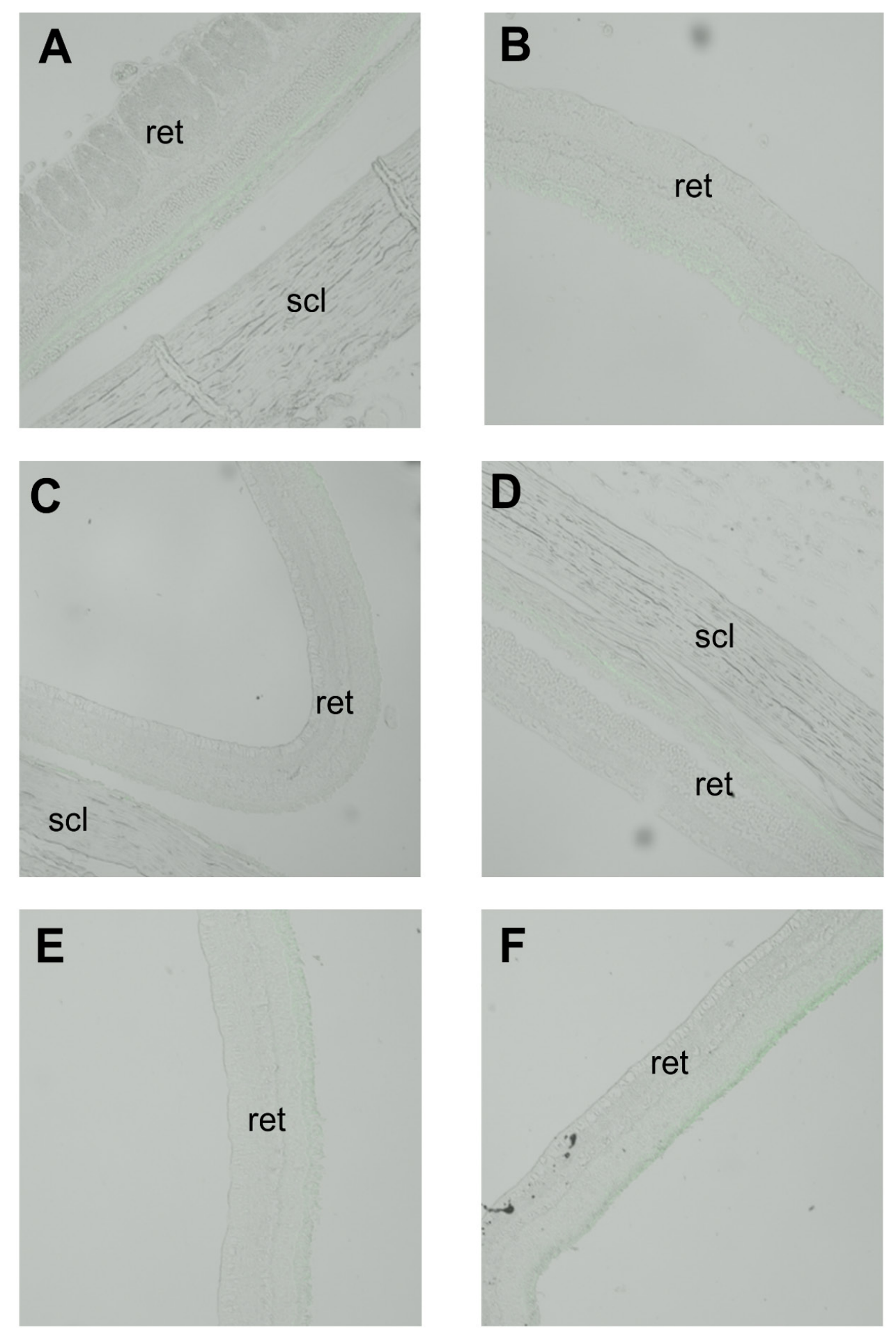

Figure 8. Sections of the retina and sclera around the posterior pole of the rabbit eyes. In all panels the retina is marked "ret" and the sclera is marked "scl". (A) bevacizumab, free in gel (rabbit 1, left eye), (B) bevacizumab, in liposomes (rabbit 1, right eye), (C) negative control (rabbit 2, left eye), (D) ranibizumab, free in gel (rabbit 2, right eye), (E) verteporfin, direct injection into vitreous chamber (rabbit 3, left eye), (F) verteporfin, in liposomes (rabbit 3, right eye).

interial cavitation is the usual explanation for the enhanced delivery. This is also the mechanism attributed to enhanced permeability of the skin barriers due to ultrasound-induced sonophoresis (3). Such intensities also have the potential for damage to surrounding materials and tissues, since it has been reported that the intensities at which maximum enhancement of skin permeability was induced by low-frequency ultrasound $\left(14 \mathrm{~W} / \mathrm{cm}^{2}\right.$ for $20 \mathrm{kHz}$ and $17 \mathrm{~W} / \mathrm{cm}^{2}$ for $40 \mathrm{kHz}$ ) also caused pitting damage of aluminium foil due to the cavitation. Indeed, intensities of low-frequency ultrasound $(23 \mathrm{kHz})$ above the cavitation threshold degraded aqueous polyvinyl alcohol (PVA) polymer (8).

This study reports a low-frequency ultrasound system in which the transducer is in direct contact with a polymer gel 
that contains the molecule or nanoparticle to be delivered. The results of the in vitro experiments indicate that the power required to enhance the diffusion in the polymer gel was only 0.2 $\mathrm{W} / \mathrm{cm}^{2}$. This is much less than the power required for previous low-frequency ultrasound systems, and below the cavitation threshold. The low power required for the system described in this report suggest that the mechanism for the enhanced diffusion is not due to cavitation. A possible explanation for the enhanced diffusion of this system reported here is due to directed Brownian motion, in an analogous way to a ratchet motor. The basis for this hypothesis is that the agarose is a physical gel with some level of crystalline structure and a level of viscoelasticity. The gold nanoparticles for the in vitro experiments were homogenously dispersed within the "tracer-gel". The low-frequency ultrasound, at a low power, could impart some momentum to the gold nanoparticles that would then cause a small motion of the nanoparticle. Clearly, the combination of the agarose concentration (to alter the polymeric backbone structure and the viscoelasticity of the gel), the mass of the nanoparticle, the power and the frequency of the ultrasound all combine to limit the range of such an imparted motion of the nanoparticle. Nonetheless, at a critical combination of low-frequency ultrasound at low power to induce a low acoustic pressure there will be sufficient time for the nanoparticle to be "pushed" through the agarose gel structure. The distance "pushed" will also be influenced by the viscoelastic forces in the agarose gel, which could serve to damp the ultrasound-imparted momentum and movement of the nanoparticles. Taken together, the results shown in Figs. 2, 3 and 6 support this interpretation of the potential mechanism of the enhanced diffusion measured with this low-frequency direct-contact ultrasound system.

The in vivo experiments with the rabbit eyes demonstrated a biotechnology application for this principle of a low-frequency and low-power ultrasound system to enhance the diffusion of therapeutic molecules. The thickness of the sclera at the limbal region of the rabbit eye (less than $0.5 \mathrm{~mm}$ ) was well-suited to the demonstrated range of nanoparticle motion (up to $1 \mathrm{~mm}$ ) from the in vitro experiments. Moreover, the therapeutic molecules were large antibodies and antibody fragments (up to $149 \mathrm{kDa}$ ), either incorporated directly into the agarose "tracer-gel" or encapsulated in liposomes that were then incorporated into the agarose "tracer-gel". The therapeutic molecules were detected in the outer retina, which demonstrated a delivery system for the eye that utilized advantages of the low-frequency and low-power ultrasound system to transport the molecules into the uveal blood circulation for subsequent delivery to the outer retina. In a previous study, low-frequency $(40 \mathrm{kHz})$ ultrasound at a low power $\left(0.12 \mathrm{~W} / \mathrm{cm}^{2}\right)$ was demonstrated to enhance the transport of a solution of dextran-FITC $(70 \mathrm{kDa})$ into the eye of a rabbit (10). That previous study supports the results shown in this present report, whereby the principle of utilising low-frequency and low-power ultrasound has potential biotechnology applications for delivery of therapeutically relevant molecules to tissues other than the skin barrier. The skin barrier appears to require higher power at low frequency to induce cavitation and sonophoresis for enhanced permeability and delivery of molecules (3).

\section{Conclusions}

This report demonstrates that low-frequency ultrasound $(40 \mathrm{kHz})$ at a low power $\left(0.2 \mathrm{~W} / \mathrm{cm}^{2}\right)$ is capable of enhancing the diffusion of gold nanoparticles in an agarose gel (of concentration $0.5 \% \mathrm{w} / \mathrm{v}$ ). The enhanced diffusion was stimulated by an ultrasound transducer in direct contact with the agarose gel that contained the gold nanoparticles. Such a direct contact allowed for low power to be generated to stimulate the diffusion, and this power was below the threshold for cavitation. This principle of ultrasound-stimulated diffusion in an agarose gel was demonstrated in a biological application for the delivery of therapeutic molecules to the outer retina of rabbit eyes.

\section{Conflicts of Interest and Acknowledgements}

The author declares he is a co-founder of a startup company, Seagull Technology Pty Ltd that develops drug-delivery systems for the eye. The procedures and care of the rabbits were approved by the RNSH/UTS Animal Care and Ethics Committee (Approval RNS/ UTS 0711-046A).

\section{References}

1. Lavon I, Kost J. Mass transport enhancement by ultrasound in non-degradable polymeric controlled release systems. J Contr Rel 1998; 54(1): 1-7.

2. Aschkenasy C, Kost J. On-demand release by ultrasound from osmotically swollen hydrophobic matrices. J Contr Rel 2005; 110 : 58- 66.

3. Terahara T, Mitragotri S, Kost J, Langer R. Dependence of low-frequency sonophoresis on ultrasound parameters; distance of the horn and intensity. Int J Pharm 2002; 235(1-2): 35-42.

4. Birkin PR, Offin DG, Vian CJB, Leighton TG. Multiple observations of cavitation cluster dynamics close to an ultrasonic horn tip. J Acoust Soc Amer 2011; 130(5): 3379-3388.

5. Leighton TG, White PR, Morfey CL, Clarke JWL, Heald GJ, Dumbrell $\mathrm{HA}$, Holland KR. The effect of reverberation on the damping of bubbles. J Acoust Soc Amer 2002; 112: 1366-1376.

6. Manaspon C, Hernandez C, Nittayacharn P, Jeganathan S, Nasongkla N, Exner AA. Increasing Distribution of Drugs Released from In Situ Forming PLGA Implants Using Therapeutic Ultrasound. Ann Biomed Eng 2017; 45(12): 2879-2887.

7. Jiang H, Tovar-Carrillo K2, Kobayashi T. Ultrasound stimulated release of mimosa medicine from cellulose hydrogel matrix. Ultrason Sonochem 2016; 32: 398-406.

8. Grönroos A, Pirkonen P, Heikkinen J, Ihalainen J, Mursunen H, Sekki H. Ultrasonic depolymerization of aqueous polyvinyl alcohol. Ultrason Sonochem 2001; 8(3): 259-264.

9. Shahar J, Avery RL, Heilweil G, Barak A, Zemel E, Lewis GP, Johnson PT, Fisher SK, Perlman I, Loewenstein A. Electrophysiologic and retinal penetration studies following intravitreal injection of bevacizumab (Avastin). Retina 2006; 26(3): 262-269.

10. Suen W-LL, Wong HS, Yu Y, Lau LCM, Lo AC-Y, Chau Y. Ultrasound mediated transscleral delivery of macromolecules to the posterior segment of rabbit eye in vivo. Invest Ophthalmol Vis Sci 2013; 56: 4358-4365. 